\title{
Exosomal miRNA-128-3p from mesenchymal stem cells of aged rats regulates osteogenesis and bone fracture healing by targeting Smad5
}

\author{
Tao Xu+, Yongjun Luo ${ }^{\dagger}$, Jiaxing Wang ${ }^{\dagger}$, Ning Zhang, Changjiang Gu, Linwei Li, Dingfei Qian, Weihua Cai", \\ Jin Fan ${ }^{*}$ and Guoyong Yin ${ }^{*}$
}

\begin{abstract}
Transplantation of mesenchymal stem cells (MSCs) has been considered an effective therapeutic treatment for a variety of diseases including bone fracture. However, there are associated complications along with MSCs transplantation. There is evidence to show that exosomes (Exos) derived from MSCs exert a similar paracrine function. In addition, repair capabilities of MSCs decline with age. Hence, this study aims to confirm whether the Exos protective function on osteogenic differentiation and fracture healing from aged MSCs was attenuated. This information was used in order to investigate the underlying mechanism. MSCs were successfully isolated and identified from young and aged rats, and Exos were then obtained. Aged-Exos exhibited significantly attenuated effects on MSCs osteogenic differentiation in vitro and facture healing in vivo. Using miRNA array analysis, it was shown that miR-128-3p was markedly upregulated in Aged-Exos. In vitro experiments confirmed that Smad5 is a direct downstream target of miR-128-3p, and was inhibited by overexpressed miR-128-3p. A series gain- and loss- function experiment indicated that miR128-3P serves a suppressor role in the process of fracture healing. Furthermore, effects caused by miR-128-3P mimic/ inhibitor were reversed by the application of Smad5/siSmad5. Taken together, these results suggest that the therapeutic effects of MSCs-derived Exos may vary according to differential expression of miRNAs. Exosomal miR-128-3P antagomir may act as a promising therapeutic strategy for bone fracture healing, especially for the elderly.
\end{abstract}

Keywords: Exosomes, miR-128-3P, Smad5, Mesenchymal stem cells, Bone fracture

\section{Introduction}

Fractures are one of the most common injuries of the musculoskeletal system, [1, 2]. However, approximately $5-10 \%$ of patients suffered from delayed or non-healing resulting from the impaired fracture repair $[3,4]$. Prolonged healing and ongoing treatment results in an undue economic burden on patients as well as poor life quality. Fracture healing is a complicated process, which is influenced by many factors [5]. Several cell types are involved

\footnotetext{
*Correspondence: caiwhspine@sina.com; fanjin@njmu.edu.cn; guoyong_ yin@sina.com

†Tao Xu, Yongjun Luo and Jiaxing Wang contributed equally to this work Department of Orthopaedics, The First Affiliated Hospital of Nanjing

Medical University, Nanjing 210029, Jiangsu, China
}

in the dynamic modeling and remodeling process of bone tissue [6]. Among all the potential factors involved in bone metabolism, the differentiation capacity of bone marrow-derived mesenchymal stem cells (MSCs) is recognized to play quite a critical role [7-9]. Lineage specific differentiation of bone marrow stem cells is crucial to tissue repair. Bone tissue regeneration requires coordinated enrollment of osteoblasts and osteoclasts that are terminally differentiated from MSCs. In particular, the osteoblast, of mesenchymal origin, is pivotal during bone remodeling and skeletal development [10]. Transplantation of MSCs into target tissues has been proven effective in many disease models, including the promotion of osteogenesis in fractures [11, 12]. However, direct transplanting of MSCs has non-negligible limitations, such as 
low survival rate, immune rejection and inducing tumor formation.

Exosomes (Exos) are 50-100 nm diameter extracellular vesicles secreted by many cell types. Exos originate from invagination of endosomal membranes and are released into the extracellular space [13]. Exosomes participate in intercellular communication by transport of functional biomolecules, such as proteins, cytokines, mRNAs, and micro-RNAs, which differ depending on the producer cells. Specific surface ligands make it possible for Exos to combine with target cells, deliver contents, and eventually produce specific biological effects [14]. Exos may be considered as an important paracrine secretion of cells [15]. Furuta T et al. [16] has demonstrated that MSCsderived exosomes could promote fracture healing in mice, which suggests that Exos transplantation may serve as a prospective substitutional strategy for MSC-related treatment in conditions of bone non-union or delayed fracture healing.

Micro-RNAs (miRNAs) are a class of highly conserved small noncoding RNAs that regulate gene expression post-transcriptionally. Micro-RNAs modulate a series of biologic processes involved in tumor progression, metabolic diseases, stem cell self-renewal, development, differentiation, and growth $[15,17-19]$. Increasing studies have found that miRNAs may function in the induction of osteoblast differentiation and bone formation. For example, Li et al. [20] showed that miRNA-21-5p promoted osteoblast differentiation in a hypoxic environment. Likewise, Xue et al. [21] suggested that miR-125b could suppress osteoblastic differentiation through an NKIRAS2 and NF- $k B$ pathway. Taken together, exploring the underlying mechanism of osteoblast differentiation induced by Exos-delivered miRNA may help us to better understand bone loss diseases and thereby develop new treatment strategies.

Aging has been correlated with bone loss and delayed fracture healing [22]. Age-related osteoporosis is associated with significantly reduced bone formation resulting from decreased numbers and degraded osteogenic ability of MSCs [23, 24]. Nevertheless, the specific age-related mechanism of these observations remains unclear. Using miRNA array analysis, we found that miR-128-3p was one of the top miRNAs in Aged-Exos. Interestingly, the expression levels of miR-128-3p were significantly higher than in Young-Exos. We predicted the downstream target genes of miR-128-3p using Targetscan database and found several potential targets which might affect fracture healing. Among these candidate genes, Smad5 (Smad family member 5), as a downstream signal molecule of bone morphogenetic proteins (BMPs), was of particular interest. BMPs are osteogenic genes that have been shown to exhibit the strongest osteogenic activity in vivo and in vitro $[25,26]$. Expression of Smad5 parallels changes in expression of endogenous BMP activity [27]. We found in this study that the protective effects of exosomes derived from aged rats' MSCs on fracture healing in vivo and osteogenic differentiation in vitro were attenuated by upregulated miR-128-3p. These findings may indicate a novel mechanism of MSCs-derived exosomes that affects bone fracture healing and provide a promising avenue for fracture treatment, especially for the elderly.

\section{Materials and methods \\ Animals}

All rats in this study were acquired from the Laboratory Animal Research Center of Nanjing Medical University. Three-month-old male Sprague-Dawley (SD) rats were used for the femoral fracture model. Four-week-old and 72-week-old SD rats were used for MSCs isolation, representing young and aged rats, respectively. This study was approved by Animal Experimentation Ethics Committee of Nanjing Medical University and all the procedures were conducted abiding the Guidelines for the Care and Use of Laboratory Animals.

\section{Cell culture}

Rat MSCs were obtained from young (4-week-old) and old (72-week-old) rats' bone marrow and seeded in $100 \mathrm{~mm}$-diameter cell culture dishes with $10 \mathrm{ml}$ specific culture medium at $37{ }^{\circ} \mathrm{C}$ with $5 \% \mathrm{CO}_{2}$. To ensure exosomes-free culture media, exosome-depleted fetal bovine serum (FBS)-containing medium was used (EXOFBS-250A-1; System Biosciences, Mountain View, CA, USA). The MSCs were washed with PBS after $48 \mathrm{~h}$ and passaged at $80 \%$ confluency and all experiments were conducted using the $3 \mathrm{rd}-5$ th passage.

\section{Identification of MSCs}

The morphology of aged and young MSCs was observed via microscopy (Axio Observer 3.1, Zeiss, Oberkochen, Germany) and the immunophenotypes of MSCs (CD90/ CD44/CD45-/CD34-) were characterized by LSR II flow cytometry (BD Bioscience). The data were analyzed by Flowjo X 10.0 (Tree Star Inc.). All experiments were conducted in triplicate. MSCs osteogenic differentiation were measured via Alkaline Phosphatase (ALP) staining and Alizarin Red staining. Toluidine Blue staining and Oil Red O staining were conducted for the identification of chondrogenic and adipogenic differentiation. For identification of MSCs differentiation, cells were induced for at least 14 days with different treatment. Then cells were fixed in $0.4 \%$ formaldehyde (Klinipath, Olen, Belgium) for $5 \mathrm{~min}$, washed with PBS and deionized water, and stained with $2 \%$ Alizarin Red solution, 0.5\% Toluidine 
Blue solution or saturated Oil Red O solution for $10 \mathrm{~min}$, then rinsed with distilled water. ALP activity was evaluated using an ALP staining kit (Beyotime Institute of Biotechnology, China). The osteogenic differentiation and mineralization level of MSCs was evaluated according to the intensity of ALP and Alizarin Red staining.

\section{Exosome isolation and purification}

MSCs were incubated for $48 \mathrm{~h}$ after reaching $80 \%$ confluency, replacing the media with exosome-depleted FBS. Then, the supernatant was collected and centrifuged successively at $300 \times g$ for $10 \mathrm{~min}$ and $2,000 \times g$ for $10 \mathrm{~min}$ at $4{ }^{\circ} \mathrm{C}$. After centrifugation, the cell supernatant was filtered through a $0.22-\mu \mathrm{m}$ sterile filter (Steritop ${ }^{\mathrm{TM}}$ Millipore, Burlington, MA) and applied to the upper compartment of an Amicon Ultra-15 Centrifuge Filter Unit (Millipore). Then the supernatant was centrifuged at $4,000 \times g$ till the volume decreased to $200 \mu \mathrm{l}$ in the upper compartment. The ultra-filtered supernatant was then washed twice with PBS and re-filtered to another $200 \mu \mathrm{l}$. The liquid was loaded onto the top of a $30 \%$ sucrose/ D2O cushion in a sterile Ultra-Clear ${ }^{\mathrm{TM}}$ tube (Beckman Coulter, Asphalt, CA, USA), followed by centrifugation at $100,000 \times g$ for $60 \mathrm{~min}$ at $4{ }^{\circ} \mathrm{C}$ with an optima L-100 $\mathrm{XP}$ Ultracentrifuge (Beckman Coulter). Exosomes were stored at $-80^{\circ} \mathrm{C}$.

\section{Exosome identification}

The morphology of the Exos was observed under a transmission electron microscope (TEM; Tecnai 12, Philips, The Netherlands). The diameter distributions of both Young-Exos and MSC-Exos were analyzed via Nanosight LM10 System (Nanosight Ltd, Navato, CA). Specific exosome surface markers like CD81 and CD63 were examined using LSR II flow cytometer (BD Bioscience) and the data were also analyzed by Flowjo X 10.0 (Tree Star Inc.).

\section{Osteogenic differentiation of MSCs}

Osteogenic differentiation of MSCs isolated from 4-week-old rats was performed $24 \mathrm{~h}$ after the incubation of Exos or transfection of miRNA mimics or inhibitors. Briefly, the original medium was replaced by osteogenesis-induction medium (20 mM $\beta$-glycerophosphate, $50 \mathrm{mM} \mathrm{L}$-ascorbic acid-2-phosphate, and $1 \mathrm{nM}$ dexamethasone in complete medium). The induction medium was refreshed every $72 \mathrm{~h}$ together with 200ul YoungExos $(200 \mu \mathrm{g} / \mathrm{mL})$, Aged-Exos $(200 \mu \mathrm{g} / \mathrm{mL})$ or PBS. The mimics or inhibitor transfections were performed after the induction medium was refreshed. Total RNA was extracted at 7 and 14 days after induction for qRT-PCR analysis.

\section{Fracture model experiments}

Rat femoral fracture model was conducted as follows. Briefly, rats were generally anesthetized before we made a 2-cm incision. A 1-mm-diameter Kirschner's wire was inserted into the femur through the patellar tendon, then a middle femoral fracture was conducted using a bone forceps. After surgery, the rats were randomly assigned to 3 groups: PBS, Young-Exos or Aged-Exos $(n=12 /$ group). PBS $(200 \mu \mathrm{L})$, Young-Exos or Aged-Exos $(200 \mu \mathrm{g}$ exosomes total protein settled in $200 \mu \mathrm{L}$ of PBS) were respectively injected around the fracture area. The entire fracture model was finished after the closure and suture of the incision. For the first 3 days post-operatively, pain levels of the rats were controlled by injecting buprenorphine every day. All efforts were made to help rats suffer the least and all surgical procedures above-mentioned were performed in a sterile environment.

\section{Microcomputed tomography (micro-CT) imaging}

Four rats from each group were randomly sacrificed at 2, 3 and 4 weeks after the surgery respectively. Femurs were removed and fixed in $4 \%$ paraformaldehyde for $24 \mathrm{~h}$. Fracture callus was evaluated using a micro-CT system (SkyScan 1172, Bruker, Belgium) at a resolution of $9 \mu \mathrm{m}$ with $50 \mathrm{kV}$ and $200 \mu \mathrm{A}$. We constructed 3-dimensional structures and analyzed the mineralized callus volume $(\mathrm{CV})$ as bone morphometric parameter. The ratio of bone volume (BV) and total volume (TV) was also calculated after 4 weeks. Then the femurs were decalcified in $10 \%$ ethylenediaminetetraacetic acid (EDTA) for 3-4 weeks, and embedded in paraffin.

\section{Western blotting analysis}

The cultured MSCs were lysed with RIPA lysis and extraction buffer (KeyGEN Biotechnology, China) to acquire bone-related proteins such as Collagen I (Col I), Alkaline phosphatase (ALP) and Runt-related transcription factor 2 (Runx2). The concentration of these proteins was determined via the bicinchoninic acid (BCA) protein quantification kit (Pierce, Waltham, MA, USA). Equal amounts of proteins were separated on an $10 \%$ SDS-PAGE gel by electrophoresis, and then transferred to poly-vinylidene fluoride (PVDF) membranes (EMD Millipore Corp., Burlington, MA). The membranes were blocked with bovine serum albumin $(5 \%, \mathrm{v} / \mathrm{v})$ for $2 \mathrm{~h}$ at room temperature prior to incubating overnight at $4{ }^{\circ} \mathrm{C}$ in respective primary Abs. After washing with Tris-Buffered Saline Tween-20 (TBST), membranes were incubated in appropriate secondary Abs for $2 \mathrm{~h}$ at room temperature. The membrane was washed with TBST after incubation, then washed and reacted with a chemi-luminescent substrate according to the manufacturer's instructions 
(Thermo Scientific, Waltham, MA, USA). The reacting bands were visualized using ECL reagent (Thermo Fisher Scientific) and imaged using ImageJ (National Institutes of Health, Bethesda, MD, USA).

The glyceraldehyde-3-phosphate dehydrogenase (GAPDH) was used as an internal control. The primary antibodies used in the experiments were anti-ALP (1:1000; Abcam, USA), anti-Runx2 (1:1000; Abcam, USA), anti-Col I (1:1000; Abcam, USA), anti-Smad5 (1:1000; Abcam, USA) and anti-GAPDH (1:1000; Abcam, USA).

\section{Quantitative real-time PCR (qRT-PCR)}

Total RNA was isolated from exosomes or cells induced for 7 and 14 days using Trizol reagent (Invitrogen, Carlsbad, CA, USA). Callus tissues collected from fracture sites at 14, 21 and 28 days post-operation were also used to extract RNA with the same Trizol reagent. A Reverse Transcription System (Toyobo, Osaka, Japan) was used to reverse-transcribe messenger RNA to complementary DNA (cDNA) and qRT-PCR was performed with SYBR Green PCR master mix (Applied Biosystems, Foster City, CA) on an ABI 7900 fast real-time PCR system (Applied Biosystems, Carlsbad, USA). GAPDH or U6 were used as an internal control to normalize the expression levels of mRNA or miRNA. The relative expression levels were then calculated using the $2^{-\Delta \Delta C T}$ method. Bulge-loop ${ }^{\text {TM }}$ miRNA qRT-PCR Primer Sets (one RT primer and a pair of qPCR primers for each set) specific for miR-128-3p is designed by RiboBio Co, Ltd. (Guangzhou, China). The primers for U6, GAPDH, ALP, Col I and Runx2 were purchased from RiboBio (Guangzhou, China) and the specific primer sequences were showed in Table 1.

\section{miRNA expression and transfection}

miR-128-3p-mimics, miR-128-3p-mimics-NC (NC-mimics), miR-128-3p-inhibitor and miR-128-3p-inhibitor-NC (NC-inhibitor) were constructed by RiboBio (Guangzhou, China) for the MSCs osteogenic differentiation assay in vitro. The MSCs were transfected with mimics or inhibitors at a density of $4 \times 10^{5}$ each well in six-well plates using Lipofectamine 3000 reagent (Invitrogen). miR-128-3p-antagomir and miR-128-3p-antagomir-NC (NC-antagomir) were constructed by RiboBio (Guangzhou, China) to treat young or aged rats' femoral fracture. We injected $20 \mathrm{nmol}$ antagomir around the fracture for each rat on day 1 post-operation and then every 3 days until euthanasia.

\section{miRNA array analysis}

To quantify miRNA in Exos derived from MSCs of young and aged rats (Young-Exos and Aged-Exos), microarray analysis was conducted with mirVana miRNA Bioarray
Table 1 The primers used for PCR

\begin{tabular}{lll}
\hline Gene name & Primer $\left(\mathbf{5}^{\prime}\right.$ to $\left.\mathbf{3}^{\prime}\right)$ & Primer sequence \\
\hline U6 & Forward & CTCGCTTCGGCAGCACA \\
& Reverse & AACGCTTCACGAATTTGCGT \\
Runx2 & Forward & CGAATAACAGCACGCTATTAA \\
& Reverse & GTCGCCAAACAGATTCATCCA \\
ALP & Forward & TAGTGAAGAGACCCAGGCGCT \\
& Reverse & ATAGGCCTCCTGAAAGCCGA \\
Coll & Forward & CCAGAA GAACTGGTACATCAGCAA \\
& Reverse & CGCCATACT CGAACTGGAATC \\
GAPDH & Forward & TCATGGGTGTGACCATGAGAA \\
& Reverse & GGCATGGACTGTGGTCATGAG \\
Smad5 & Forward & CGGTAGCCAACTGACTTTGAGT \\
& Reverse & ACCTTGTTTCCAGCCCAACA \\
\hline
\end{tabular}

V9.2 (Ambion). miRNA was purified from $22 \mu \mathrm{g}$ total RNA obtained from Young-Exos or Aged-Exos using the flash PAGE system (Ambion) in triplicate. Samples were analyzed according to methods previously described [28]. With the Microarray Data Analysis Tool (Filgen, Inc.), the miRNA array data were normalized using global normalization. The selection criteria of miRNA referred to the results based on miRNA array PCR data.

\section{Luciferase reporter assay}

Wild-type Smad5 luciferase reporter vectors (Wt-Smad5) were constructed by cloning the fragment of Smad5 into the downstream region of the renilla psiCHECK2 vector (Promega, WI, USA). The seed region of Smad5 was mutated (Mut- Smad5) to remove the complementarity to miR-128-3p in order to generate the Smad5 mutant reporter. Wt-Smad5 or Mut- Smad5 was transfected into HEK293T cells (ATCC, USA) together with miR-128-3p mimics or miR-128-3p inhibitor. Luciferase activity was measured $48 \mathrm{~h}$ post-transfection with the Dual Luciferase Reporter Assay System (Promega) and normalized to the firefly luciferase activity.

\section{Immunohistochemistry}

Femurs were fixed in 4\% paraformaldehyde and embedded with paraffin. After endogenous peroxides and proteins were blocked, $6-\mu \mathrm{m}$-thick sections were incubated with primary anti-Smad5 antibody (Abcam, Cambridge, UK) overnight at $4{ }^{\circ} \mathrm{C}$. Sections were incubated with secondary antibody at $37{ }^{\circ} \mathrm{C}$ for $1 \mathrm{~h}$ and with 3,3-diaminobenzidine (DAB) for $3 \mathrm{~min}$.

\section{Statistical analysis}

All independent experiments were performed in triplicate and all quantitative data were expressed as 
mean \pm SD. Statistical analysis was performed via GraphPad software 7.0 and SPSS 19.0. Two-tailed Student's $\mathrm{t}$-test was used for comparisons between two groups and One-Way Analysis of Variance (ANOVA) with Bonferroni post hoc test were used to calculate the P-values for multiple comparisons. A value of $\mathrm{P}<0.05$ was considered as statistically significant and represented as the symbols “*”.

\section{Results}

Identification of MSCs

MSCs were obtained from femurs of 4-week-old and 72-week-old rats as described above. MSCs were identified by morphology and flow cytometry at passage number three. Cells exhibited a spindle-like shape (Fig. 1a) but aged MSCs had a flattened shape compared to young MSCs. The chondrogenic, adipogenic and osteogenic differentiation potential of MSCs were verified with Toluidine Blue, Oli Red $\mathrm{O}$, and ALP staining, respectively (Fig. 1b). Flow cytometry analysis confirmed that MSCs were positive for CD44 and CD90, but negative for CD34 or CD45 (Fig. 1c, d).

\section{Characterization of exosomes}

The Exos were absorbed by MSCs as showed in Additional file 1: Figure S1. Other characterization includes morphological observation by TEM, dynamic light scattering (DLS) analysis, and Flow Cytometry analysis. As Fig. 2a shows, the morphology of Young-Exos showed a rounded shape under TEM, which resembled the morphology of Aged-Exos. The diameter size of these particles was evaluated by DLS analysis, both ranging from 50 to $150 \mathrm{~nm}$ (Fig. 2b). Specific surface markers of exosomes, including CD63 and CD81 was detected to be positive in Exos by Flow Cytometry analysis (Fig. 2c). These findings were similar to previous studies [29-31] and indicated a successful isolation and identification of the exosomes derived from young and aged MSCs.

\section{Young-Exos promote MSCs osteogenesis differentiation in vitro}

The next step was to investigate whether Exos induced from young and aged MSCs have therapeutic effects on cell osteogenesis differentiation in vitro. MSCs of 4-weekold rats were cultured using osteogenic differentiation induction medium together with $200 \mu \mathrm{g} / \mathrm{mL}$ Aged-Exos, Young-Exos, or equal quantities of PBS, respectively. The expression of osteogenesis-related genes (Runx2, ALP and Col I) were examined every seven days using qRTPCR. As shown in Fig. 3a, b, the highest mRNA expression levels of Runx2, ALP and Col I was found in the Young-Exos group, suggesting a remarkable increase in osteogenic ability of MSCs incubated with Young-Exos.
Both the ALP and Alizarin Red staining showed the strongest intensity in MSCs after 7 or 14 days of incubation with Young-Exos (Fig. 3c, d).

\section{Exosome uptake experiment}

Dil solution (Molecular Probes, Eugene, OR, USA) was added to the solution contained Young-Exos or AgedExos (1: 200) and incubated at $4{ }^{\circ} \mathrm{C}$ for $15 \mathrm{~min}$. Then adding PBS and ultra-centrifugate as mentioned above to remove excess dye. Repeat this step three times. Fluorescently labeled Young-Exos and Aged-Exos were obtained and then respectively co-cultured with MSCs for $24 \mathrm{~h}$, fixed with $4 \%$ paraformaldehyde for $15 \mathrm{~min}$, rinsed 3 times by PBS. Finally, the uptake of exosomes was observed with laser confocal microscopy.

\section{Bone fracture healing in rats after Exos application}

As demonstrated above, Young-Exos were found to accelerate MSCs osteogenesis differentiation in vitro. Our micro-CT examination analyzed different callus formations after application of PBS, Aged-Exos, or Young-Exos. In Fig. 4a, apparent fracture gaps were observed 2 weeks postoperatively in all the groups but callus volumes were found larger in the Young-Exos group compared to the other two groups. Similar results were detected 3 and 4 weeks postoperative. In the Young-Exos group, fracture gaps were covered by a callus, and volumes of newly formed calluses were larger as well. According to the results from Micro-CT examination, transplantation of Young-Exos increased the CV significantly (Fig. 4b). Micro-CT reconstruction on post-operative day 28 showed that BV/TV was highly elevated in rats treated with Young-Exos (Fig. 4c). As showed in Fig. 4d, the expression level of Runx2, ALP and Col I in calluses were upregulated as well after administration of Young-Exos after 2, 3 and 4 weeks.

\section{The miR-128-3p is upregulated in Aged-Exos}

It was shown that Young-Exos enhanced osteogenesis in vitro and promoted fracture healing in vivo when compared to Aged-Exos and PBS controls. It has been concluded that exosomal miRNAs regulate multiple biological functions functioning in intercellular communication [32-34]. Therefore, Exos were collected from the supernatant of aged and young MSCs and the miRNA levels were evaluated by miRNA array analysis. Heat map analysis (Fig. 5a) showed that miR-128-3p was upregulated in Aged-Exos. The top ten miRNAs in Aged-Exos were selected and qRT-PCR was conducted to confirm the miRNA levels. Figure $5 c$ showed that miR-128-3p was the most upregulated miRNAs in the Aged-Exos compared to the Young-Exos. 


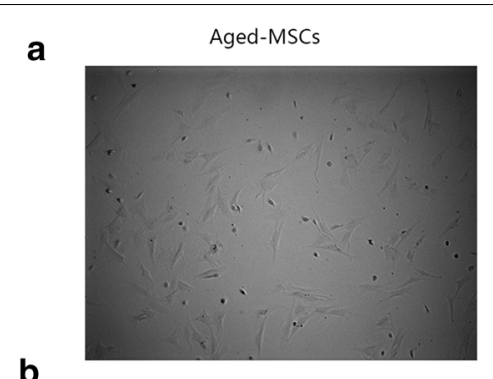

b
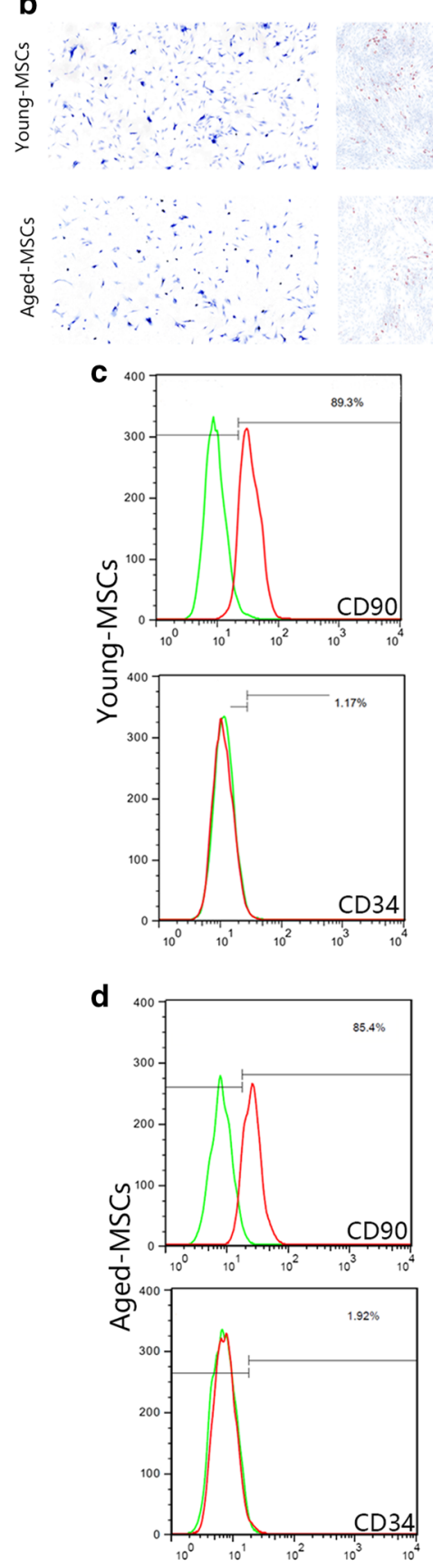

Young-MSCS
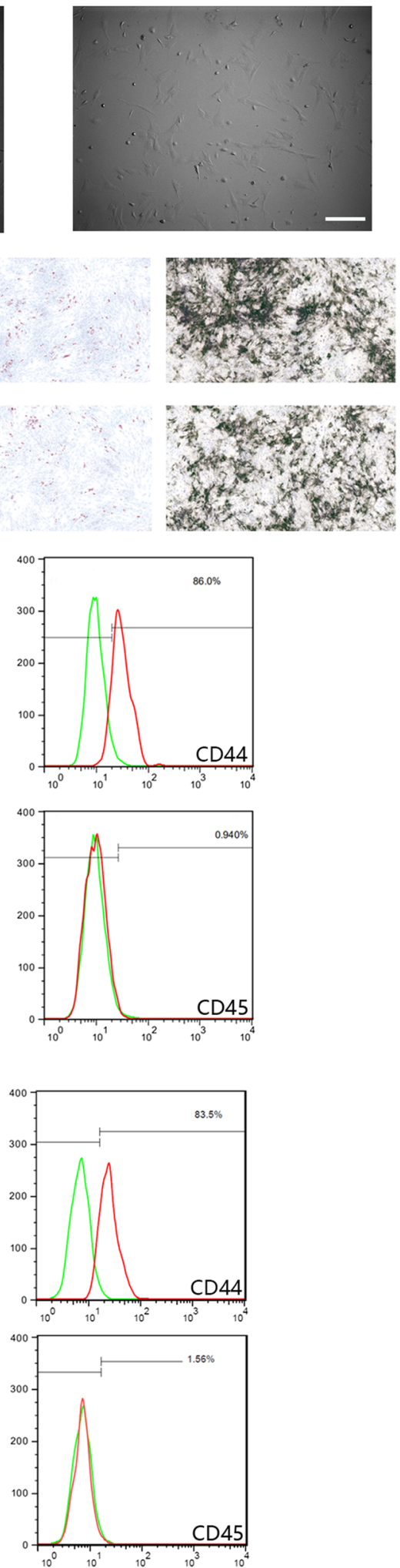

Fig. 1 a MSCs adopted a spindle-like morphology, whereas aged MSCs demonstrated a flattened morphology compared to young MSCs (scale: $100 \mu \mathrm{m})$. b Toluidine Blue, Oli Red O and ALP staining of MSCs (scale: $100 \mu \mathrm{m}$ ). c, $\mathbf{d}$ MSCs were positive for CD44 and CD90 but negative for CD34 or CD45 
a

Young-Exos

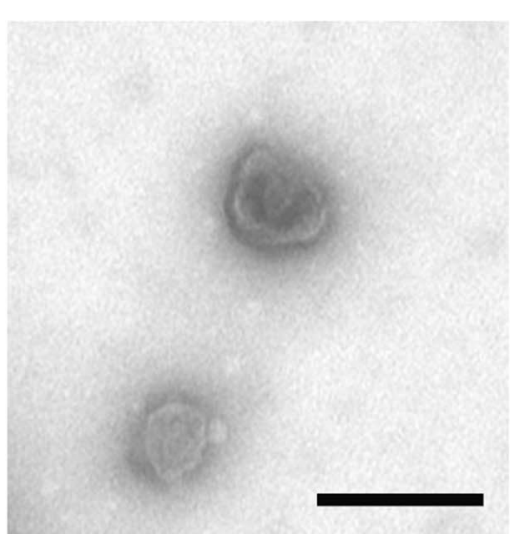

b

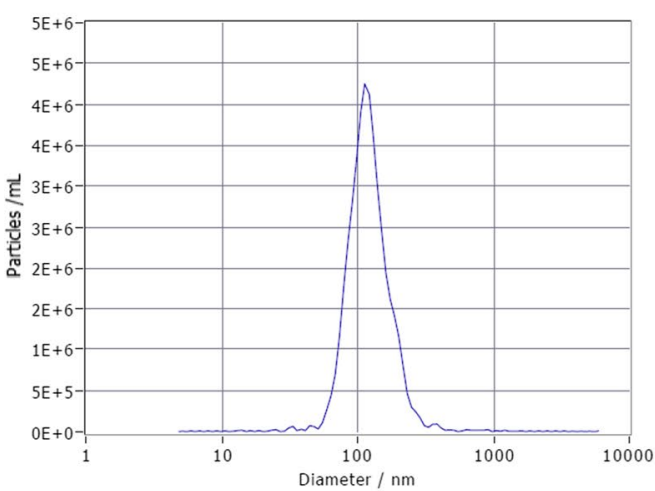

C
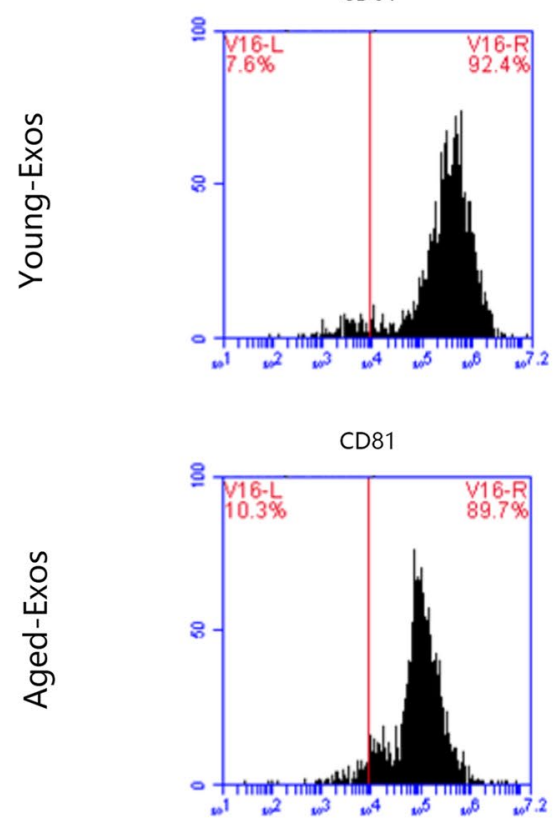

Aged-Exos

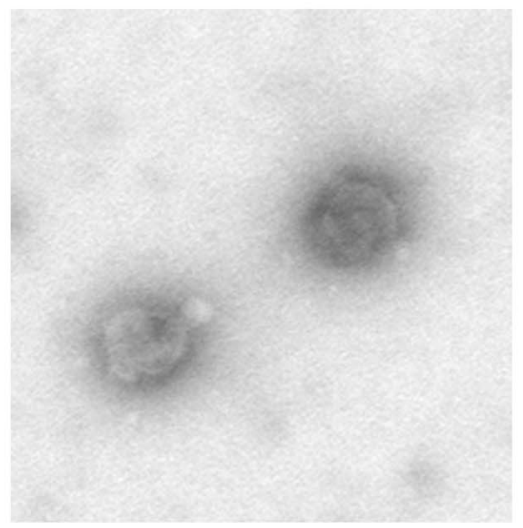

Aged-Exos

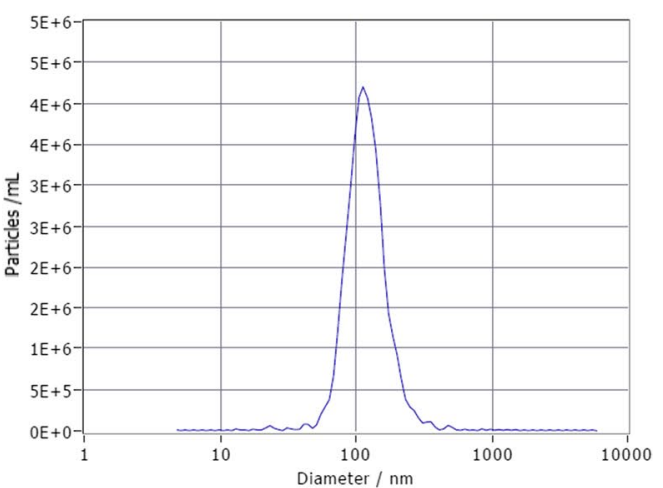

CD63

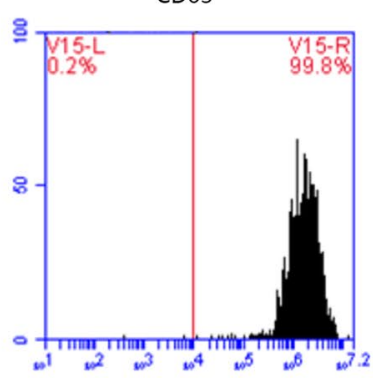

CD63

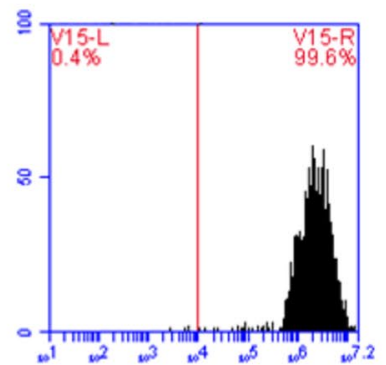

Fig. 2 a The morphology of Young-Exos and Aged-Exos (scale: 100 nm). b The diameter of Young-and Aged-Exos, ranging from 50 to $150 \mathrm{~nm}$. c Specific surface markers of exosomes, including CD63 and CD81 
a

$7 d$

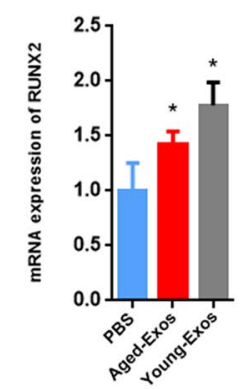

b

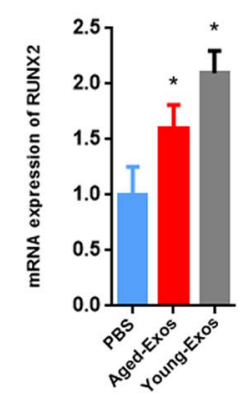

C

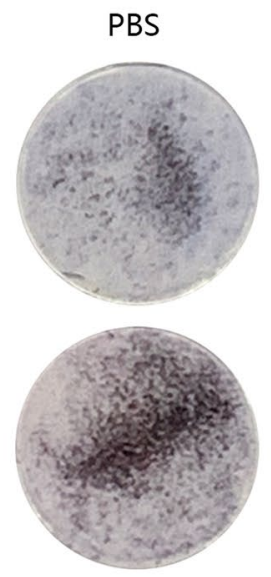

d

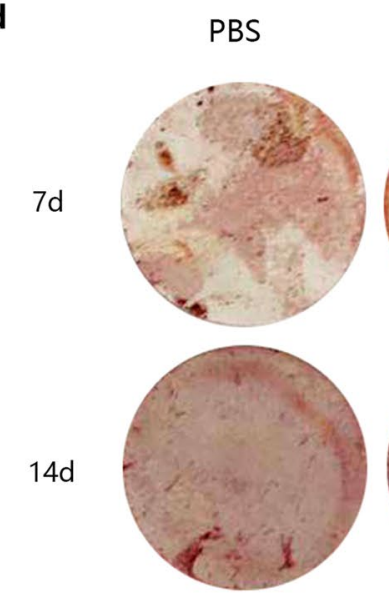

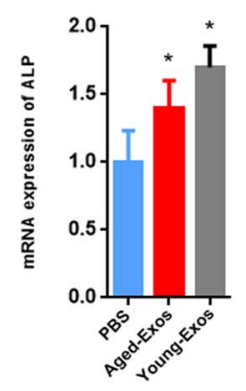
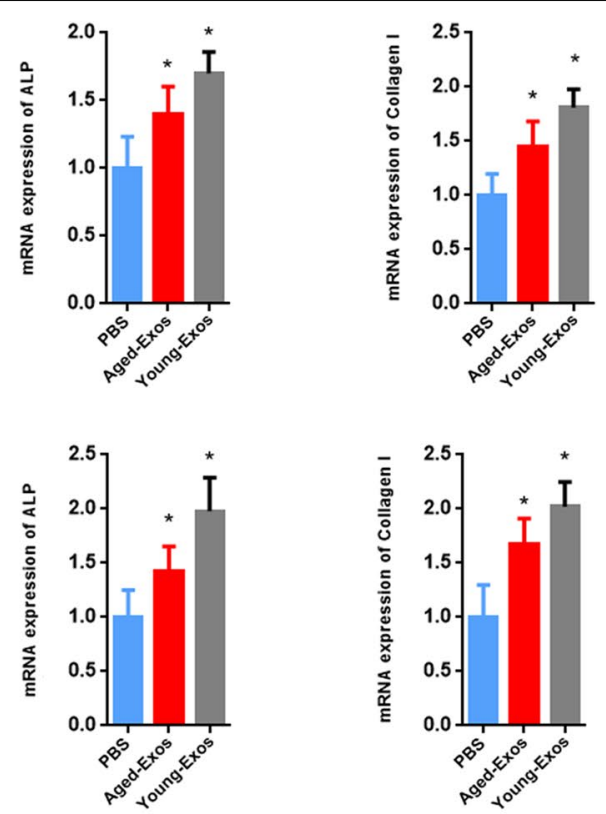

Aged-Exos
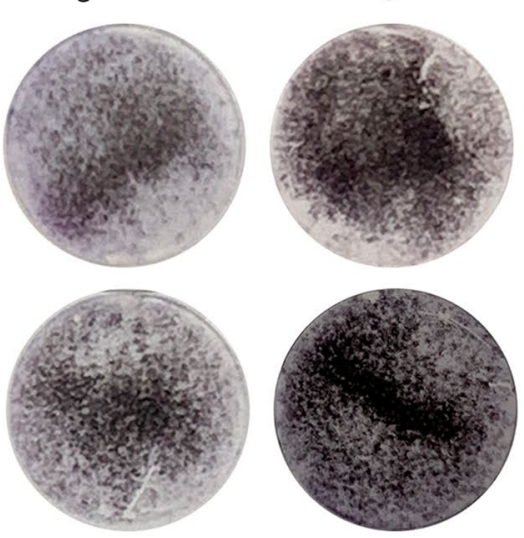

Aged-Exos

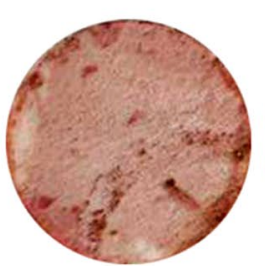

Young-Exos
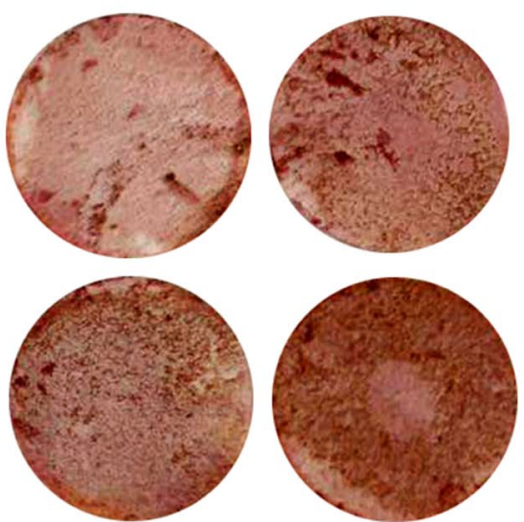

Fig. 3 a, b The mRNA expression levels of Runx2, ALP and Col I ( $n=5$, PBS vs Age-Exos, $P<0.05$, Age-Exos vs Young-Exos, $P<0.05)$. $\mathbf{c}, \mathbf{d}$ ALP staining and Alizarin Red staining after 7 or 14 days of incubation with Exos 


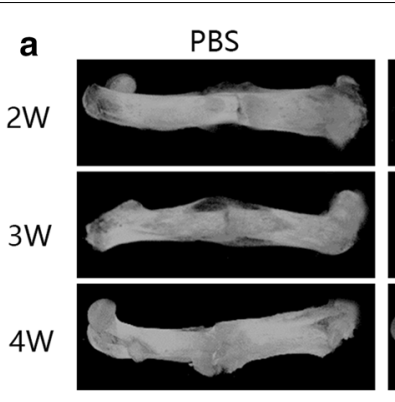

b

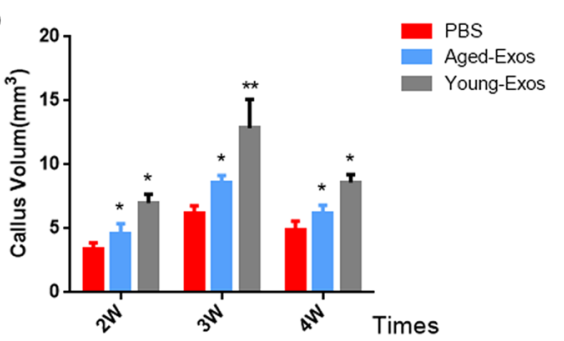

d

$2 \mathrm{~W}$
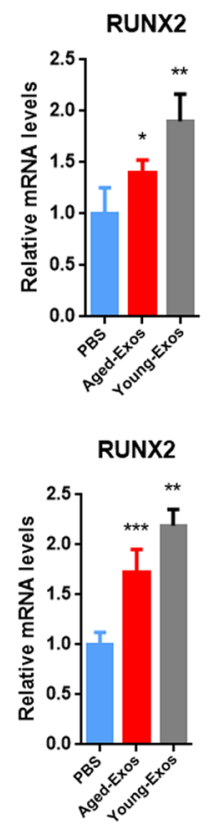

RUNX2

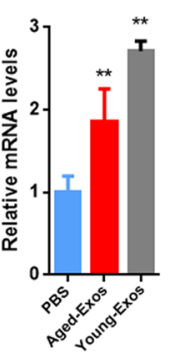

Aged-Exos
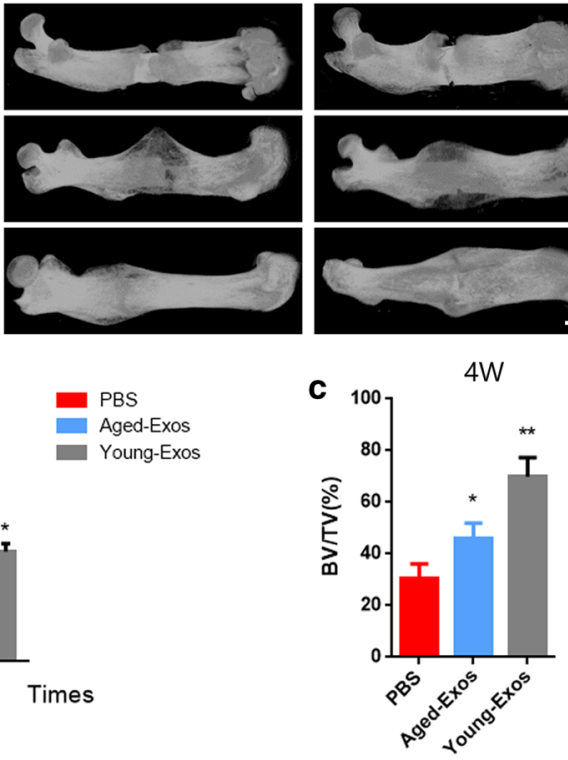

ALP
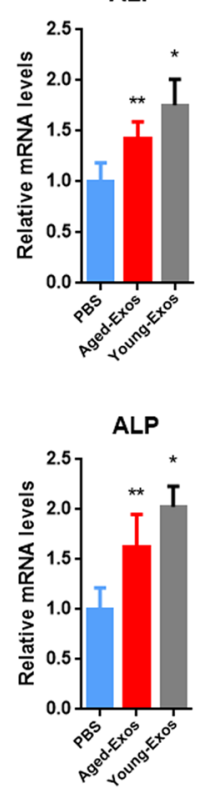

ALP

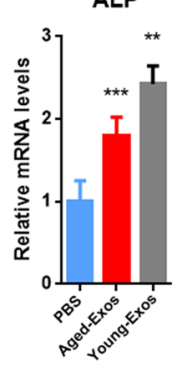

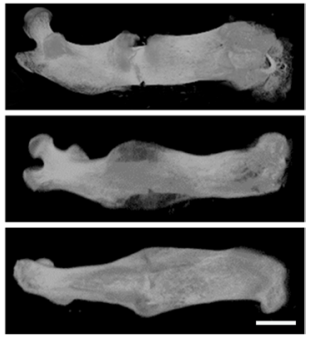

Collagen I
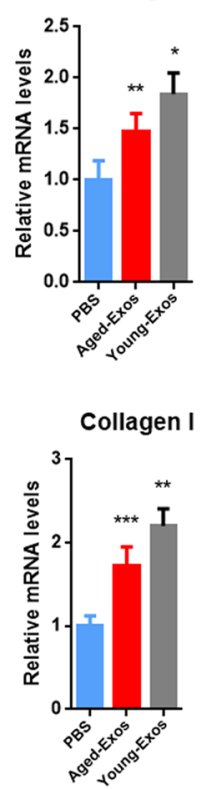

Collagen I

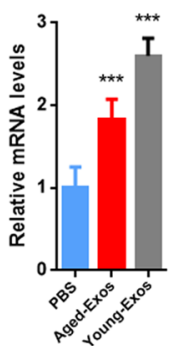

Young-Exos

Fig. 4 a, b Micro-CT images and Callus volumes (scale: $1 \mathrm{~mm}$ ). c BV/TV on day 28. d The mRNA levels of Runx2, ALP and Collagen I in calluses ( $\mathrm{n}=4$ PBS vs Age-Exos, $P<0.05$, Age-Exos vs Young-Exos, $\mathrm{P}<0.05$ ) 


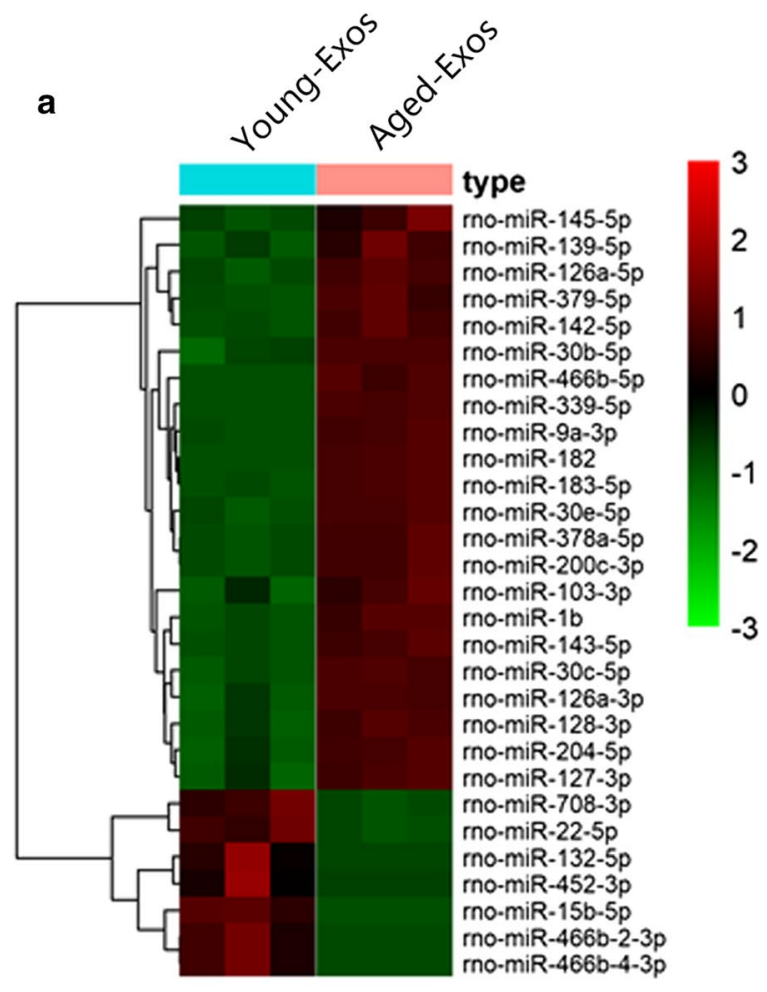

b
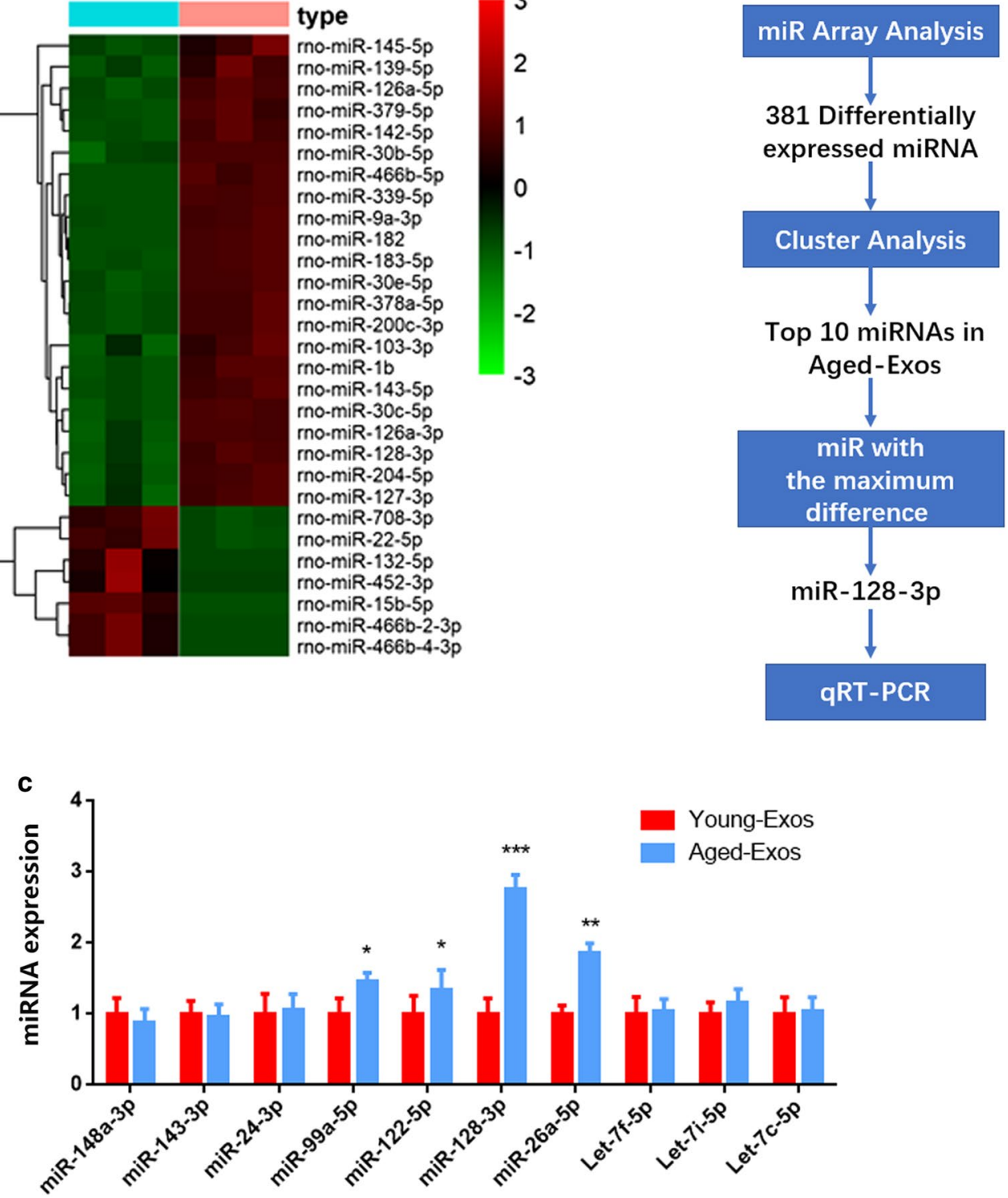

Fig. 5 a The heat map showed that miR-128-3p was upregulated in Aged-Exos. b The schematic diagram of microRNA screening and selection. $\mathbf{c}$ qRT-PCR showed that miR-128-3p was the most upregulated miRNAs in the Aged-Exos among the top 10 miRNAs

\section{Exosomal miR-128-3p regulates Smad5 by directly targeting the $3^{\prime}-$ UTR.}

Since the miR-128-3p expression was confirmed as abundant in Aged-Exos, the next step was to predict the potential target genes of miR-128-3p. According to predictions from the online database Targetscan, Smad5 stood out among all other candidate target genes. Smad5 is one of the downstream genes of BMPs and has paralleled expression levels with endogenous BMP activity.
The BMP/Smad axis functions in cell osteogenesis and osseous development of multiple cell and tissue types. Next, the relationship between exosomal miR-128-3P and Smad5 was investigated using a series of in vitro experiments. The seed region of the Mut-Smad5 was reconstructed as shown in Additional file 2: Figure S2. The Wt-Smad5 or Mut-Smad5 were transfected into HEK293T cells together with miR-128-3p mimics or miR-128-3p inhibitor. The transfection efficiency of the 
mimics and inhibitor was verified by qRT-PCR (Additional file 3: Figure S3). Luciferase reporter assay was conducted to verify that the Smad5 $3^{\prime}$ UTR is a direct target for miR-128-3p. A wild-type Smad5 and mutant-type Smad5 luciferase reporter gene vector was constructed as shown in Additional file 2: Figure S2. The relative luciferase activity decreased when miR-128-3p mimics were co-transfected with Wt-Smad5, but not with the MutSmad5 (Fig. 6a). However, an enhanced luciferase activity was observed when the miR-128-3p inhibitor was cotransfected with Wt-Smad5. Using Western blotting analysis, elevated expressional levels of Smad5 were detected in MSCs after administration of the miR-128-3P inhibitors. As shown in Fig. 6b, c, after the administration of miR-128-3P inhibitor for 14 days, the protein levels of Smad5 in MSCs was markedly increased compared with levels in the NC-inhibitor group. In contrast, decreased Smad5 expression was found in MSCs incubated with the miR-128-3P mimics. In summary, we confirmed that miR-128-3P negatively modulates Smad5 by directly targeting its $3^{\prime}$-UTR.

\section{MiR-128-3P/Smad5 axis modulates osteogenic differentiation of MSCs in vitro}

In the subsequent experiments in vitro, we investigated the effects of miR-128-3P and Smad5 on osteogenic differentiation ability of young and aged MSCs respectively. Firstly, we upregulated miR-128-3P level in young MSCs and a significant decrease of Runx2, ALP and Col I were detected by the Western blotting assays. Then upregulating Smad5 rescued the negative effects of MSCs osteogenic differentiation caused by the administration of miR-128-3P mimics (Fig. 6d, e). Similarly, we found that the osteogenic differentiation was accelerated when aged MSCs were incubated with miR-128-3P inhibitor and these positive effects could also be reversed by silencing Smad5 (Fig. 6f, g). For this part, we demonstrated that miR-128-3P exerted an inhibitory role on MSCs' osteogenic differentiation and these effects could be abolished by Smad5.

\section{Alternation of miR-128-3P in vivo could affect the femoral fracture healing process in rats}

In order to further investigate the function of exosomal miR-128-3P on rats' femoral fracture coalescence, both miR-128-3P antagomir and antagomir-NC were constructed by RiboBio (Guangzhou, China). Using the qRT-PCR assay, the expression of miR-128-3P in fractured calluses showed a suppression in the antagomir group at day 14 after local injection of antagomir around the fracture area (Fig. 7a). The post-operative micro-CT reconstruction images at 2, 3 and 4 weeks showed that, compared to the $\mathrm{NC}$ antagomir group, the fracture gaps were diminishing in the miR-128-3P antagomir group. Furthermore, the $\mathrm{CV}$ and the BV/TV showed a marked increased by miR-128-3P antagomir treatment as well (Fig. $7 b, c)$. According to the qRT-PCR results, the mRNA levels of ALP, Runx2, Col I and Smad5 in the callus were upregulated by miR-128-3P antagomir at day 28 (Fig. 7d). Furthermore, the results of immunohistochemistry showed that the expression level of Smad5 was significantly upregulated in rats treated with miR-128-3P antagomir for 4 weeks (Fig. 7e). These data indicated an anti-osteogenic function of miR-128-3P in vivo via targeting Smad5.

\section{Discussion}

With an aging society comes the prevalence of agerelated diseases. alone such example is increased bone fractures among the elderly. Bone fracture healing ability differs greatly depending on the age of the organism. For example, numerous elderly people suffer from delayed bone fracture healing or non-union reaching up to $18.5 \%$ in some specific types of fractures [35]. The prolonged rehabilitation process after fracture may cause $=$ severe complications and may even result in death [36-38]. Mesenchymal stem cells (MSCs) have great potential for self-replication and differentiation into several types of cells including osteoblasts [39]. Osteoblasts are of mesenchymal origin and function in skeletal development and bone formation [40, 41]. Additionally, MSCs can secrete various growth factors and angiogenic factors such as BMP-2 and vascular endothelial growth factor (VEGF) [42]. As a result, MSCs transplantation has been considered as a practical option for bone tissue engineering [43, 44]. However, the clinical application of MSCs is restricted by the invasive harvesting procedures, the dependence for carriers, as well as consideration of ethical and safety issues [45, 46]. More importantly, the biological effects of MSCs decline notably in age-related diseases. Given this situation, efficient applications of these stem cells for clinical disorders still have to cross may hurdles ahead (Additional file 4).

Exosomes (Exos) was first reported in 2010 as the active agent secreted by MSCs against myocardial ischemia reperfusion injury [47]. Afterwards, MSCderived Exos were reported to exert therapeutic effects on a variety of diseases (osteonecrosis, ischemic diseases, spinal cord injury, and chronic cutaneous wounds) $[48,49]$. Increasing evidence indicates that the reparative abilities of MSCs are derived from secretion and paracrine mechanisms [50-52]. Exos are known for their homogeneous therapeutic efficacy while overcoming the restrictions of direct cell transplantation. It has been demonstrated in several studies that Exos derived from MSCs could enhance the healing process 


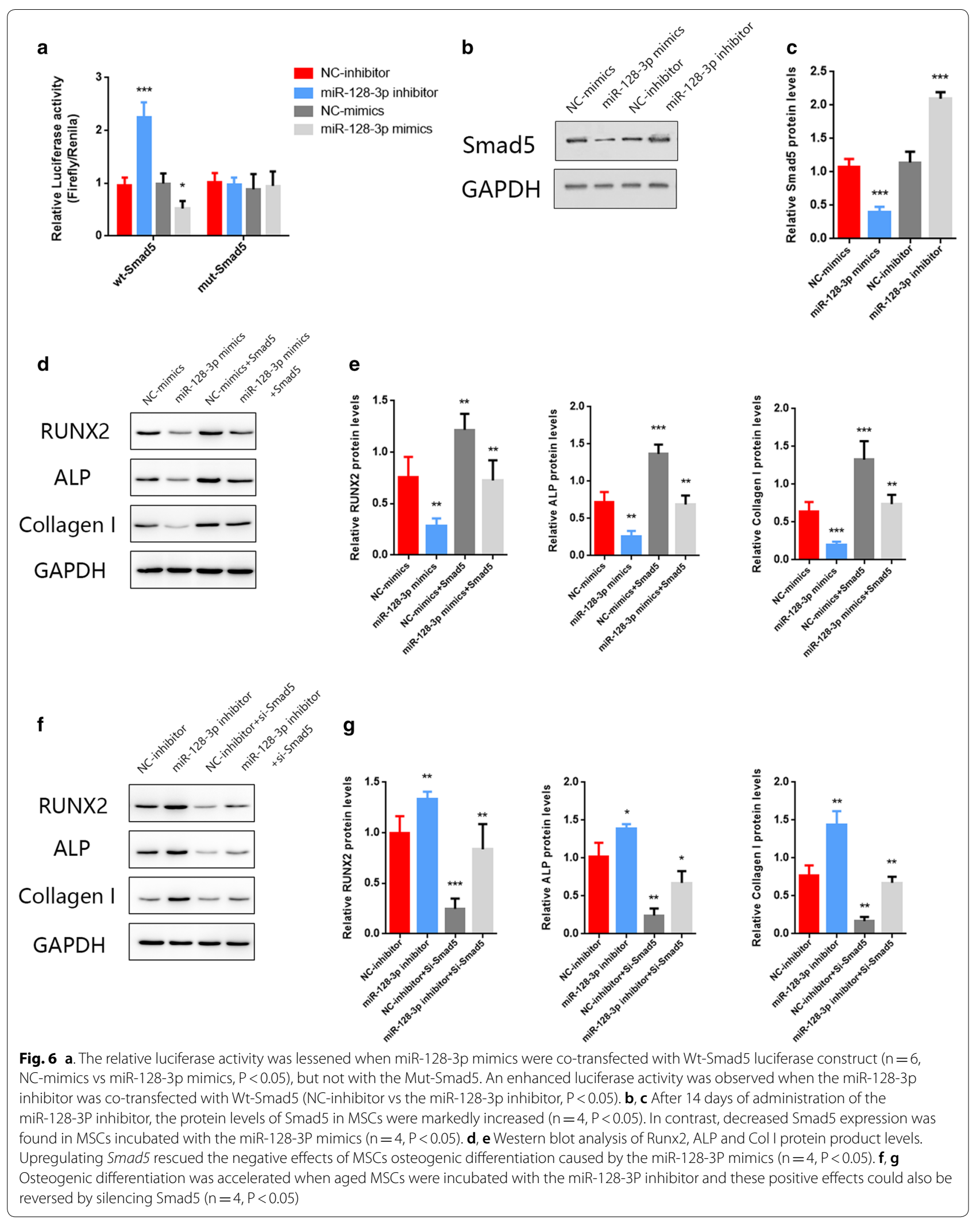


(See figure on next page.)

Fig. 7 a The expression of miR-128-3P in fractured callus at day 14 (NC-antagomir vs miR-128-3P antagomir, $P<0.05$ ). b, c The post-operative micro-CT reconstruction images at 2, 3 and 4 weeks. The CV and the BV/TV were increased by the miR-128-3P antagomir treatment $(n=4$, NC-antagomir vs miR-128-3P antagomir, $\mathrm{P}<0.05$, scale: $1 \mathrm{~mm}$ ). $\mathbf{d}$ The mRNA level of ALP, Runx2, Collagen I and Smad5 in the callus at day 28(NC-antagomir vs miR-128-3P antagomir, $\mathrm{P}<0.05$ ). e The results of immunohistochemistry (NC-antagomir vs miR-128-3P antagomir, $\mathrm{P}<0.05$, scale: $100 \mu \mathrm{m})$

in animal fractures or bone defect models $[16,53,54]$. The osteogenic differentiation ability of MSCs decays as the individuals get older. Biological information, such as proteins, mRNAs, and miRNAs contained in MSCs and MSC-derived Exos may differ due to distinct age status. In our preliminary in vivo studies, Exos derived from aged rats were observed with attenuated therapeutic function in a rat femoral fracture model. It was speculated whether Exos derived from young MSCs exerted more protective effects on the fracture healing in vivo and MSCs osteogenesis in vitro than those from aged MSCs. This was conducted in order to investigate the underlying mechanism.

Hence, in this study, several experiments were performed to verify our hypothesis. Firstly, we successfully obtained and identified MSCs from aged and young rats. It was demonstrated that both aged and young MSCs could differentiate into osteoblasts, chondrocytes, or adipocytes. The characteristics of aged MSCs resembled young MSCs with the exception of a more flattened morphology. Then, we isolated Exos from the supernatant of either aged or young MSCs. The TEM and NTA results showed high morphological similarities between the Aged-Exos and Young-Exos according to their shape, size, and density. Nevertheless, local injection treatment revealed that Young-Exos promoted fracture healing more abundantly than Age-Exos. Furthermore, in vitro studies showed that the osteogenesis of MSCs were significantly enhanced after incubation with Young-Exos. A recent study conducted by Taisuke et al. [16] demonstrated that MSCs-derived Exos promoted fracture healing in a mouse model. These Exos were secreted by MSCs isolated from young mice. This report coincided with the findings presented here. However, in the present study, we did not further investigate whether the effects of Exos would decrease with senescence of secreting cells.

Next, we sought to identify the biological macromolecules that contributed to the varied effects between Aged- or Young- Exos. Therefore, miRNA array analysis was performed and more than 100 miRNAs were found to be differentially expressed. Specifically, in Aged-Exos, the content of 84 miRNAs were twice more than those in Young-Exos, and the content of 40 miRNAs was found to be less than the half of those in Young-Exos. The top ten miRNAs contained in Aged-Exos were then listed, and it was found that miR-128-3p was most upregulated.
Next, the potential downstream target genes of miR128-3p were investigated. According to the database Targetscan, several candidate genes were reportedly associated with osteogenesis, such as BMP3, Smad9 and Smad5. With the administration of miR-128-3p inhibitor or mimics, BMP3 and smad9 results are not significant in the protein levels. In addition, Smad5 is the classical protein of osteogenic pathway. Among these genes, we took the most interest in Smad5. BMP groups were one of the three subfamilies of the transforming growth factor- $\beta$ (TGF- $\beta$ ) family $[55,56]$. The Smad $1,2,3,5$, and 8 (or 9) are collectively referred to as receptor-regulatedSmads (R-Smads). Smad1/5/8, (maybe 9) are regulated through BMP receptor complexes [56-58]. Previous studies demonstrated that BMP2/4 transmitted signals via Smad1/5/8, and Smad5 was involved in the enhancement of cellular ALP activity induced by BMP6 $[59,60]$. Furthermore, various recent studies have revealed a pivotal role of Smad5 in the osteogenesis of MSCs. Smad5 has been considered a target of several miRNAs and long noncoding RNA [61-64]. Given this observation, we sought to identify whether Smad5 was a target of miR128-3p using the Luciferase reporter assay. As shown in Fig. 6a, luciferase activity was attenuated by the cotransfection of miR-128-3p mimics and Wt-Smad5, but not with the Mut-Smad5. When Wt-Smad5 was incubated with miR-128-3p inhibitor, there was an enhanced luciferase activity. In addition, according to Western Blot results, the expression levels of Smad5 protein in MSCs was markedly upregulated after administration of miR128-3P inhibitor for 14 days. Based on these findings, it was demonstrated that miR-128-3P negatively modulates Smad 5 by directly binding its $3{ }^{\prime}$ UTRs and possibly inhibits the osteogenesis process both in vitro and in vivo.

In vivo and in vitro experiments were conducted to verify whether decreased Smad5 contributed to the delayed osteogenic process caused by upregulating miR-128-3p in Aged-Exos. Overexpressed miR-128-3p was achieved by transfection of miR-128-3p mimics into young MSCs. Then it was found that the osteogenesis-related proteins such as Runx2, Collagen I, and ALP were remarkably downregulated. This finding indicated that miR-128-3p suppressed the osteogenic ability of MSCs in vitro. However, overexpressing Smad5 could reverse the inhibitory functions caused by miR-128-3p, which means miR128-3p exerted its effects through Smad5. However, 


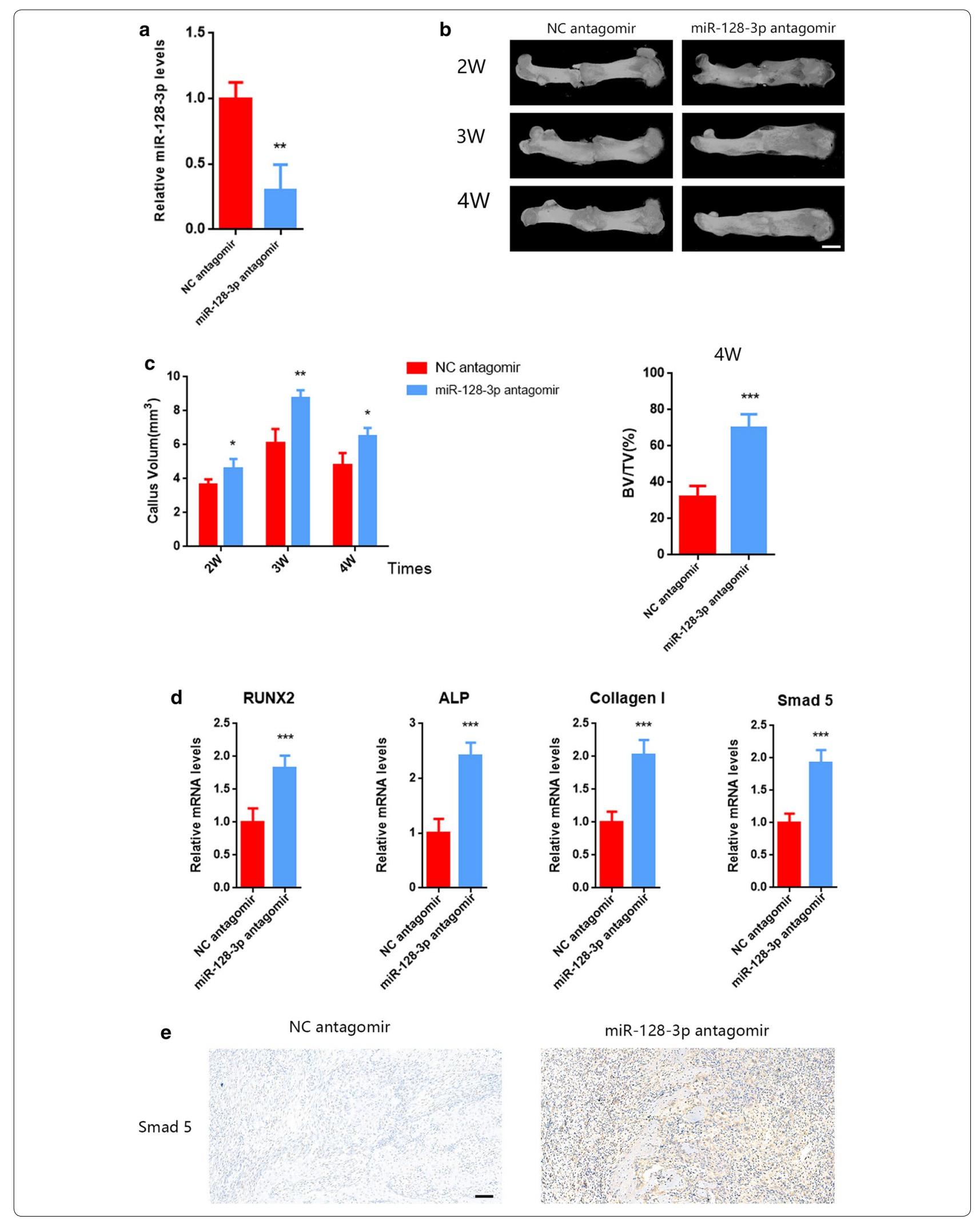


silencing Smad5 also abolished the positive results delivered by the miR-128-3p inhibitor.

The miR-128-3p antagomir was constructed to investigated the effects of miR-128-3p on fracture healing in vivo. After 2 weeks of local injections of the miR-128-3p antagomir around the surgery area, rats showed a remarkable increase in fracture healing based on micro-CT images. The expression of Smad5 underwent notable increasement in the callus tissue harvested from rats treated with miR-128-3p antagomir. Meanwhile, the immunohistochemical results of increased Smad5 in miR-128-3p antagomir group supported qRTPCR as well. All these assays confirmed a direct interaction between miR-128-3P and Smad5, and indicated their essential roles in the recovery process of fractures in vivo.

Exosomes derived from MSCs have been reported by numerous studies to exert their biological functions by delivering specific miRNAs to the target cells. A recent study showed that over-expressed miRNA-181a in MSCderived exosomes influenced the inflammatory response after myocardial ischemia-reperfusion injury [65]. Another study demonstrated that Exos derived from miR-30b-3p-overexpressing MSCs protect against acute lung injury induced by lipopolysaccharides [66]. Furthermore, miR-100-5p-abundant exosomes derived from fat pad MSCs was confirmed to protect articular cartilage via suppressing mTOR levels in osteoarthritis [67]. The miRNA array analysis presented in this report demonstrated that miR-128-3P was highly expressed in AgedExos compared to Young-Exos. In addition, miR-128-3P could be transferred to the target MSCs after incubation with Aged-Exo. Several previous studies showed that miR-128-3P played an inhibitory role in various cancer cases. For example, it was reported that miR-128-3p suppressed breast cancer progression by targeting LIM domain kinase 1(LIMK1) [68]. Furthermore, miR-128-3p was demonstrated to inhibit glioma cell proliferation and differentiation through the IRS-1/PI3K/AKT signaling pathway [69]. Exosome-transmitted miR-128-3p could also exert its anticancer effect by increasing the chemosensitivity of oxaliplatin-resistant colorectal cancer [70]. However, as far as we know, there have been no reports about the specific role of miR-128-3p in the MSCs osteogenic differentiation and bone fracture healing process. Likewise, Bing et al. [71] conducted bioinformatics and microarray analysis of miRNAs using fracture calluses from aged female mice and found several highly expressed miRNAs, including miR-128-3p. However, in this study, the effects of miR-128-3p on fracture healing was not investigated. In the present study, through a series of loss- and gain-function experiments, it was confirmed that upregulation of Smad5 in vitro or in vivo could abolish the adverse effects of miR-128-3p in the process of bone fracture healing. Smad5 was reported to take part in BMP-induced osteogenesis in various studies. Even without BMP2, Smad5 could still induce the expression of ALP and suppress myogenin-CTA activity after transfection into $\mathrm{C} 2 \mathrm{C} 12$ myoblasts [72]. Type 1 collagen (Col I), alkaline phosphatase (ALP), and Runt related gene 2 (Runx2) are the necessary transcription factors for the osteogenic process and are responsible for the activation of $\mathrm{OB}$ differentiation marker genes. Runx2 is related to BMP2 signal transduction by interacting with Smad5, and coordination with BMP2-induced Smad5 is essential for Runx 2 to activate osteogenesis [73, 74]. In our study, after the inhibition of miR-128-3p, the Smad5 expression was remarkably elevated and consequently, all these osteogenic marker genes were significantly upregulated, indicating that the osteogenesis process was enhanced.

\section{Conclusion}

In this study, we demonstrated for the first time a novel phenomenon that Aged-Exos had diminished repair ability for bone fracture in rats. It was confirmed that miR128-3p expression levels in MSCs, and their secreted Exos, augment as cell senescence arises. By suppressing Smad5 expression, the enriched levels of miR-128-3p in Aged-Exos attenuate the therapeutic effect and hold back the fracture healing process. These results not only supported the therapeutic effects of Young-Exos, but also demonstrated that Smad5 is one of the downstream target genes of exosomal miR-128-3p. There were other candidate genes that were not yet tested and verified in our study such as Smad9 or BMP3. These genes may also act as the target of miR-128-3p and may exert the synergistic effects with Smad5 on fracture healing. Thus, the antagomir for miR-128-3p may work as a novel and effective approach for bone fractures of aged patients.

\section{Supplementary information}

Supplementary information accompanies this paper at https://doi. org/10.1186/s12951-020-00601-w.

Additional file 1: Figure S1. The Exos were absorbed by MSCs (scale: 20 $\mu \mathrm{m})$.

Additional file 2: Figure S2. Wild-type Smad5 and mutant-type Smad5 luciferase reporter gene vector was constructed.

Additional file 3: Figure S3. The transfection efficiency of the mimics and the inhibitor was verified by qRT-PCR.

Additional file 4. miRNA Array Analysis of Young and Aged Exos.

\section{Abbreviations}

MSCs: Mesenchymal stem cells; Exos: Exosomes; miRNAs: Micro-RNAs; NKIRAS2: NF-KB inhibitor interacting RAS-like 2; BMPs: Bone morphogenetic proteins; Smad5: Smad family member 5; SD rats: Sprague-Dawley rats; FBS: Fetal bovine serum; ALP staining: Alkaline Phosphatase staining; micro-CT: Microcomputed tomography; CV: Callus volume; BV: Bone volume; TV: Total volume; EDTA: Ethylenediaminetetraacetic acid; Col I: Collagen I; Runx2: 
Runt-related transcription factor 2; BCA: Bicinchoninic acid; PVDF: Polyvinylidene fluoride; TBST: Tris-Buffered Saline Tween-20; GAPDH: Glyceraldehyde-3-phosphate dehydrogenase; NC-mimics: miR-128-3p-mimics-NC; NC-inhibitor: miR-128-3p-inhibitor-NC; NC-antagomir: miR-128-3p-antagomirNC; DAB: 3,3-diaminobenzidine; ANOVA: Analysis of Variance; DLS: Dynamic light scattering; VEGF: Vascular endothelial growth factor; TGF- $\beta$ : Transforming growth factor- $\beta$.

\section{Authors' contributions}

YL, GY, and JF designed research; JW and NZ analyzed data; YL, TX, JW, NZ, LL, $D Q$, and CG performed research; YL, GY, and WC wrote the paper; JF and WC contributed new reagents or analytic tools. All authors read and approved the final manuscript.

\section{Funding}

The authors acknowledge financial support from the National Natural Science Foundation of China (Grant Nos. 81772352, 81401807, 81772351, and 81271988), and Natural Science Foundation of Jiangsu Province (Grant No. BK2012876).

\section{Availability of data and materials}

Materials described in the manuscript, including all relevant raw data, will be freely available to any scientist wishing to use them for non-commercial purposes, without breaching participant confidentiality.

\section{Ethics approval and consent to participate}

All experimental procedures were conducted in conformity with institutional guidelines for the care and use of laboratory animals and protocols, which were approved by the Animal Care and Use Committee of Nanjing Medical University (No. IACUC 1601036). Sprague-Dawley male rats were provided by the Animal Experimental Center of Nanjing Medical University.

\section{Consent for publication}

The authors give their consent for information about ourselves to be published in Journal of Nanobiotechnology. We understand that the information will be published without our children or relative's name attached, but that full anonymity cannot be guaranteed. We understand that the text and any pictures or videos published in the article will be freely available on the internet and may be seen by the general public. The pictures, videos and text may also appear on other websites or in print, may be translated into other languages or used for commercial purposes. We have been offered the opportunity to read the manuscript.

\section{Competing interests}

The authors declare no conflict of interests.

Received: 11 January 2020 Accepted: 5 March 2020

Published online: 16 March 2020

\section{References}

1. Claes $L$, Recknagel $S$, Ignatius A. Fracture healing under healthy and inflammatory conditions. Nat Rev Rheumatol. 2012;8(3):133-43.

2. Einhorn TA, Gerstenfeld LC. Fracture healing: mechanisms and interventions. Nat Rev Rheumatol. 2015;11(1):45-544.

3. Gomez-Barrena E, Rosset P, Lozano D, Stanovici J, Ermthaller C, Gerbhard F. Bone fracture healing: cell therapy in delayed unions and nonunions. Bone. 2015;70:93-101.

4. 4van Griensven M. Preclinical testing of drug delivery systems to bone. Adv Drug Deliv Rev 2015; 94: 151-164.

5. Komatsu DE, Warden SJ. The control of fracture healing and its therapeutic targeting: improving upon nature. J Cell Biochem. 2010;109(2):302-11.

6. Colnot C. Skeletal cell fate decisions within periosteum and bone marrow during bone regeneration. J Bone Miner Res. 2009;24(2):274-82.

7. Dozza B, Salamanna F, Baleani M, Giavaresi G, Parrilli A, Zani L, Lucarelli E, Martini L, Fini M, Donati DM. Nonunion fracture healing: Evaluation of effectiveness of demineralized bone matrix and mesenchymal stem cells in a novel sheep bone nonunion model. J Tissue Eng Regen Med. 2018;12(9):1972-85.
8. Lin H, Sohn J, Shen H, Langhans MT, Tuan RS. Bone marrow mesenchymal stem cells: Aging and tissue engineering applications to enhance bone healing. Biomaterials. 2019;203:96-110.

9. Lin W, Xu L, Pan Q, Lin S, Feng L, Wang B, Chen S, Li Y, Wang H, Li Y, Wang Y, Lee W, Sun D, Li G. Lgr5-overexpressing mesenchymal stem cells augment fracture healing through regulation of Wnt/ERK signaling pathways and mitochondrial dynamics. FASEB J. 2019:33(7):8565-77.

10. Buettmann EG, McKenzie JA, Migotsky N, Sykes DA, Hu P, Yoneda S, Silva MJ. VEGFA From early osteoblast lineage cells (Osterix + ) is required in mice for fracture healing. J Bone Miner Res. 2019;34(9):1690-706.

11. Sinclair KL, Mafi P, Mafi R, Khan WS. The use of growth factors and mesenchymal stem cells in orthopaedics: in particular, their use in fractures and non-unions: a systematic review. Curr Stem Cell Res Ther. 2017;12(4):312-25.

12. Wang X, Wang C, Gou W, Xu X, Wang Y, Wang A, Xu W, Guo Q, Liu S, Lu Q, Meng $\mathrm{H}$, Yuan M, Peng J, Lu S. The optimal time to inject bone mesenchymal stem cells for fracture healing in a murine model. Stem Cell Res Ther. 2018;9(1):272.

13. Skotland T, Sandvig K, Llorente A. Lipids in exosomes: current knowledge and the way forward. Prog Lipid Res. 2017;66:30-41.

14. Barile L, Vassalli G. Exosomes: therapy delivery tools and biomarkers of diseases. Pharmacol Ther. 2017;174:63-78.

15. Dupont C, Kappeler L, Saget S, Grandjean V, Levy R. Role of miRNA in the transmission of metabolic diseases associated with paternal diet-induced obesity. Front Genet. 2019;10:337.

16. Furuta T, Miyaki S, Ishitobi H, Ogura T, Kato Y, Kamei N, Miyado K, Higashi Y, Ochi M. Mesenchymal stem cell-derived exosomes promote fracture healing in a mouse model. Stem Cells Transl Med. 2016;5(12):1620-30.

17. Vos PD, Leedman PJ, Filipovska A, Rackham O. Modulation of miRNA function by natural and synthetic RNA-binding proteins in cancer. Cell Mol Life Sci. 2019;76(19):3745-52.

18. Shcherbata HR. miRNA functions in stem cells and their niches: lessons from the Drosophila ovary. Curr Opin Insect Sci. 2019;31:29-36.

19. Chakraborty C, Chin KY, Das S. miRNA-regulated cancer stem cells: understanding the property and the role of miRNA in carcinogenesis. Tumour Biol. 2016;37(10):13039-488.

20. Li L, Jiang D. Hypoxia-responsive miRNA-21-5p inhibits Runx2 suppression by targeting SMAD7 in MC3T3-E1 cells. J Cell Biochem. 2019;120(10):16867-755.

21. Xue N, Qi L, Zhang G, Zhang Y. miRNA-125b Regulates Osteogenic Differentiation of Periodontal Ligament Cells Through NKIRAS2/NF-kappaB Pathway. Cell Physiol Biochem. 2018:48(4):1771-81.

22. Manolagas SC, Parfitt AM. What old means to bone. Trends Endocrinol Metab. 2010;21(6):369-74

23. Sethe S, Scutt A, Stolzing A. Aging of mesenchymal stem cells. Ageing Res Rev. 2006;5(1):91-116.

24. Fehrer $\mathrm{C}$, Laschober $\mathrm{G}$, Lepperdinger $\mathrm{G}$. Aging of murine mesenchymal stem cells. Ann N Y Acad Sci. 2006;1067:235-42.

25. Liu MZ, Zhou DC, Liu Q, Xie FL, Xiang DX, Tang GY, Luo SL. Osteogenesis activity of isocoumarin a through the activation of the PI3K-Akt/ Erk cascade-activated BMP/RUNX2 signaling pathway. Eur J Pharmacol. 2019;858:172480.

26. Ruehle MA, Krishnan L, Vantucci CE, Wang Y, Stevens HY, Roy K, Guldberg RE, Willett NJ. Effects of BMP-2 dose and delivery of microvascular fragments on healing of bone defects with concomitant volumetric muscle loss. J Orthop Res. 2019;37(3):553-61.

27. Katakawa Y, Funaba M, Murakami M. Smad8/9 Is Regulated Through the BMP Pathway. J Cell Biochem. 2016;117(8):1788-96.

28. Ishida H, Tatsumi T, Hosui A, Nawa T, Kodama T, Shimizu S, Hikita H, Hiramatsu N, Kanto T, Hayashi N, Takehara T. Alterations in microRNA expression profile in HCV-infected hepatoma cells: involvement of miR-491 in regulation of $\mathrm{HCV}$ replication via the PI3 kinase/Akt pathway. Biochem Biophys Res Commun. 2011:412(1):92-7.

29. Ge M, Ke R, Cai T, Yang J, Mu X. Identification and proteomic analysis of osteoblast-derived exosomes. Biochem Biophys Res Commun. 2015:467(1):27-322.

30. Skog J, Wurdinger T, van Rijn S, Meijer DH, Gainche L, Sena-Esteves M, Curry WJ, Carter BS, Krichevsky AM, Breakefield XO. Glioblastoma microvesicles transport RNA and proteins that promote tumour growth and provide diagnostic biomarkers. Nat Cell Biol. 2008;10(12):1470-6. 
31. De Jong OG, Van Balkom BW, Schiffelers RM, Bouten CV, Verhaar MC Extracellular vesicles: potential roles in regenerative medicine. Front Immunol. 2014;5:608.

32. Kulkarni B, Kirave P, Gondaliya P, Jash K, Jain A, Tekade RK, Kalia K. Exosomal miRNA in chemoresistance, immune evasion, metastasis and progression of cancer. Drug Discov Today. 2019;24(10):2058-67.

33. Yu X, Odenthal M, Fries JW. Exosomes as miRNA carriers: formation-function-future. Int J Mol Sci. 2016;17:12.

34. Fleshner M, Crane CR. Exosomes, DAMPs and miRNA: features of stress physiology and immune homeostasis. Trends Immunol. 2017;38(10):768-76.

35. Fong K, Truong V, Foote CJ, Petrisor B, Williams D, Ristevski B, Sprague $\mathrm{S}$, Bhandari M. Predictors of nonunion and reoperation in patients with fractures of the tibia: an observational study. BMC Musculoskelet Disord. 2013;14:103.

36. Hernandez RK, Do TP, Critchlow CW, Dent RE, Jick SS. Patient-related risk factors for fracture-healing complications in the United Kingdom General Practice Research Database. Acta Orthop. 2012;83(6):653-60.

37. Parker MJ, Raghavan R, Gurusamy K. Incidence of fracture-healing complications after femoral neck fractures. Clin Orthop Relat Res. 2007;458:175-9.

38. Castillo RC, Bosse MJ, MacKenzie EJ, Patterson BM. Impact of smoking on fracture healing and risk of complications in limb-threatening open tibia fractures. J Orthop Trauma. 2005;19(3):151-7.

39. Niemeyer P, Szalay K, Luginbuhl R, Sudkamp NP, Kasten P. Transplantation of human mesenchymal stem cells in a non-autogenous setting for bone regeneration in a rabbit critical-size defect model. Acta Biomater. 2010;6(3):900-8.

40. Komori T. Regulation of osteoblast differentiation by transcription factors. J Cell Biochem. 2006;99(5):1233-9.

41. Karsenty G, Kronenberg HM, Settembre C. Genetic control of bone formation. Annu Rev Cell Dev Biol. 2009;25:629-48.

42. Pittenger MF, Mackay AM, Beck SC, Jaiswal RK, Douglas R, Mosca JD, Moorman MA, Simonetti DW, Craig S, Marshak DR. Multilineage potential of adult human mesenchymal stem cells. Science. 1999;284(5411):143-7.

43. Horwitz EM, Prockop DJ, Fitzpatrick LA, Koo WW, Gordon PL, Neel M, Sussman M, Orchard P, Marx JC, Pyeritz RE, Brenner MK. Transplantability and therapeutic effects of bone marrow-derived mesenchymal cells in children with osteogenesis imperfecta. Nat Med. 1999;5(3):309-13.

44. Song H, Tao L, Wang F, Wang W, Wei Y, Shen W, Zhou F. Effect of bone mesenchymal stem cells transplantation on the micro-environment of early osteonecrosis of the femoral head. Int J Clin Exp Pathol. 2015;8(11):14528-34.

45. Katsara O, Mahaira LG, Iliopoulou EG, Moustaki A, Antsaklis A, Loutradis D, Stefanidis K, Baxevanis CN, Papamichail M, Perez SA. Effects of donor age, gender, and in vitro cellular aging on the phenotypic, functional, and molecular characteristics of mouse bone marrow-derived mesenchymal stem cells. Stem Cells Dev. 2011;20(9):1549-61.

46. Tasso R, Ulivi V, Reverberi D, Lo SC, Descalzi F, Cancedda R. In vivo implanted bone marrow-derived mesenchymal stem cells trigger a cascade of cellular events leading to the formation of an ectopic bone regenerative niche. Stem Cells Dev. 2013;22(24):3178-91.

47. Lai RC, Arslan F, Lee MM, Sze NS, Choo A, Chen TS, Salto-Tellez M, Timmers L, Lee CN, El OR, Pasterkamp G, de Kleijn DP, Lim SK. Exosome secreted by MSC reduces myocardial ischemia/reperfusion injury. Stem Cell Res. 2010;4(3):214-22.

48. Mendt M, Rezvani K, Shpall E. Mesenchymal stem cell-derived exosomes for clinical use. Bone Marrow Transplant. 2019;54(Suppl 2):789-92.

49. Toh WS, Lai RC, Hui J, Lim SK. MSC exosome as a cell-free MSC therapy for cartilage regeneration: Implications for osteoarthritis treatment. Semin Cell Dev Biol. 2017;67:56-64.

50. Toh WS, Foldager CB, Pei M, Hui JH. Advances in mesenchymal stem cellbased strategies for cartilage repair and regeneration. Stem Cell Rev Rep. 2014;10(5):686-96.

51. Hofer HR, Tuan RS. Secreted trophic factors of mesenchymal stem cells support neurovascular and musculoskeletal therapies. Stem Cell Res Ther. 2016;7(1):131

52. Watson L, Elliman SJ, Coleman CM. From isolation to implantation: a concise review of mesenchymal stem cell therapy in bone fracture repair. Stem Cell Res Ther. 2014;5(2):51.
53. Qi X, Zhang J, Yuan H, Xu Z, Li Q, Niu X, Hu B, Wang Y, Li X. Exosomes Secreted by Human-Induced Pluripotent Stem Cell-Derived Mesenchymal Stem Cells Repair Critical-Sized Bone Defects through Enhanced Angiogenesis and Osteogenesis in Osteoporotic Rats. Int J Biol Sci. 2016;12(7):836-49.

54. Qin Y, Wang L, Gao Z, Chen G, Zhang C. Bone marrow stromal/stem cell-derived extracellular vesicles regulate osteoblast activity and differentiation in vitro and promote bone regeneration in vivo. Sci Rep. 2016:6:21961.

55. Miyazono K, Kamiya Y, Morikawa M. Bone morphogenetic protein receptors and signal transduction. J Biochem. 2010;147(1):35-51.

56. Sakaki-Yumoto M, Katsuno Y, Derynck R. TGF-beta family signaling in stem cells. Biochim Biophys Acta. 2013;1830(2):2280-96.

57. Zi Z, Chapnick DA, Liu X. Dynamics of TGF-beta/Smad signaling. FEBS Lett. 2012;586(14):1921-8.

58. Massague J. TGFbeta signalling in context. Nat Rev Mol Cell Biol. 2012;13(10):616-30.

59. Lee JS, Kim ME, Seon JK, Kang JY, Yoon TR, Park YD, Kim HK. Bone-forming peptide-3 induces osteogenic differentiation of bone marrow stromal cells via regulation of the ERK1/2 and Smad1/5/8 pathways. Stem Cell Res. 2018;26:28-35

60. Li A, Xia X, Yeh J, Kua H, Liu H, Mishina Y, Hao A, Li B. PDGF-AA promotes osteogenic differentiation and migration of mesenchymal stem cell by down-regulating PDGFRalpha and derepressing BMP-Smad1/5/8 signaling. PLOS ONE. 2014;9(12):e113785.

61. Zhang W, Chen L, Wu J, Li J, Zhang X, Xiang Y, Li F, Wu C, Xiang L, Ran Q, Li Z. Long noncoding RNA TUG1 inhibits osteogenesis of bone marrow mesenchymal stem cells via Smad5 after irradiation. Theranostics. 2019;9(8):2198-208.

62. Mencia Cl, Curtin CM, Duffy GP, O'Brien FJ. Harnessing an Inhibitory Role of miR-16 in Osteogenesis by Human Mesenchymal Stem Cells for Advanced Scaffold-Based Bone Tissue Engineering. Tissue Eng Part A. 2019;25(1-2):24-33.

63. Wei F, Yang S, Guo Q, Zhang X, Ren D, Lv T, Xu X. MicroRNA-21 regulates Osteogenic Differentiation of Periodontal Ligament Stem Cells by targeting Smad5. Sci Rep. 2017;7(1):16608.

64. Gu Y, Ma L, Song L, Li X, Chen D, Bai X. miR-155 Inhibits Mouse Osteoblast Differentiation by Suppressing SMAD5 Expression. Biomed Res Int. 2017;2017:1893520

65. Wei Z, Qiao S, Zhao J, Liu Y, Li Q, Wei Z, Dai Q, Kang L, Xu B. miRNA-181a over-expression in mesenchymal stem cell-derived exosomes influenced inflammatory response after myocardial ischemia-reperfusion injury. Life Sci. 2019;232:116632.

66. Yi X, Wei X, Lv H, An Y, Li L, Lu P, Yang Y, Zhang Q, Yi H, Chen G. Exosomes derived from microRNA-30b-3p-overexpressing mesenchymal stem cells protect against lipopolysaccharide-induced acute lung injury by inhibiting SAA3. Exp Cell Res. 2019;383(2):111454.

67. Wu J, Kuang L, Chen C, Yang J, Zeng WN, Li T, Chen H, Huang S, Fu Z, Li J, Liu R, Ni Z, Chen L, Yang L. miR-100-5p-abundant exosomes derived from infrapatellar fat pad MSCs protect articular cartilage and ameliorate gait abnormalities via inhibition of mTOR in osteoarthritis. Biomaterials. 2019;206:87-100

68. Zhao J, Li D, Fang L. MiR-128-3p suppresses breast cancer cellular progression via targeting LIMK1. Biomed Pharmacother. 2019;115:108947.

69. Huo L, Wang B, Zheng M, Zhang Y, Xu J, Yang G, Guan Q. miR-128-3p inhibits glioma cell proliferation and differentiation by targeting NPTX1 through IRS-1/PI3K/AKT signaling pathway. Exp Ther Med. 2019:17(4):2921-30.

70. Liu T, Zhang X, Du L, Wang Y, Liu X, Tian H, Wang L, Li P, Zhao Y, Duan W, Xie Y, Sun Z, Wang C. Exosome-transmitted miR-128-3p increase chemosensitivity of oxaliplatin-resistant colorectal cancer. Mol Cancer. 2019;18(1):43.

71. He B, Zhang ZK, Liu J, He YX, Tang T, Li J, Guo BS, Lu AP, Zhang BT, Zhang G. Bioinformatics and microarray analysis of miRNAs in aged female mice model implied new molecular mechanisms for impaired fracture healing. Int J Mol Sci. 2016:17:8.

72. Fujii M, Takeda K, Imamura T, Aoki H, Sampath TK, Enomoto S, Kawabata M, Kato M, Ichijo H, Miyazono K. Roles of bone morphogenetic protein type I receptors and Smad proteins in osteoblast and chondroblast differentiation. Mol Biol Cell. 1999;10(11):3801-13. 
73. Vimalraj S, Arumugam B, Miranda PJ, Selvamurugan N. Runx2: Structure, function, and phosphorylation in osteoblast differentiation. Int J Biol Macromol. 2015;78:202-8.

74. Lee KS, Kim HJ, Li QL, Chi XZ, Ueta C, Komori T, Wozney JM, Kim EG, Choi $J Y$, Ryoo HM, Bae SC. Runx2 is a common target of transforming growth factor beta1 and bone morphogenetic protein 2, and cooperation between Runx2 and Smad5 induces osteoblast-specific gene expression in the pluripotent mesenchymal precursor cell line C2C12. Mol Cell Biol. 2000;20(23):8783-92.

\section{Publisher's Note}

Springer Nature remains neutral with regard to jurisdictional claims in published maps and institutional affiliations.
Ready to submit your research? Choose BMC and benefit from:

- fast, convenient online submission

- thorough peer review by experienced researchers in your field

- rapid publication on acceptance

- support for research data, including large and complex data types

- gold Open Access which fosters wider collaboration and increased citations

- maximum visibility for your research: over $100 \mathrm{M}$ website views per year

At BMC, research is always in progress.

Learn more biomedcentral.com/submissions 